\title{
Role of neutrophil extracellular traps in chronic kidney injury induced by bisphenol-A
}

\author{
Shiyun Tong1,*, Shumin Yang1,*, Ting Li2,*, Rufei Gao³, Jinbo Hu¹, Ting Luo1, Hua Qing1, Qianna Zhen¹, Renzhi Hu1, \\ Xuan Li1, Yi Yang1, Chuan Peng4,† and Qifu Li1,†

\begin{abstract}
1Department of Endocrinology, The First Affiliated Hospital of Chongqing Medical University, Chongqing, China 2Department of Endocrinology, The First Affiliated Hospital of Chengdu Medical College, Chengdu, China

${ }^{3}$ School of Public Health and Management, Chongqing Medical University, Chongqing, China

${ }^{4}$ The Chongqing Key Laboratory of Translational Medicine in Major Metabolic Diseases, The First Affiliated Hospital of Chongqing Medical University, Chongqing, China
\end{abstract}

Correspondence should be addressed to C Peng or Q Li: pengchuan2096@sina.com or liqifu@yeah.net

*(S Tong, S Yang and T Li are co-first authors)

${ }^{\dagger}$ (C Peng and Q Li are co-corresponding authors)

\begin{abstract}
Bisphenol-A (BPA) is a common environmental pollutant, and exposure to it is associated with proteinuria and may predict the progression of chronic kidney disease; however, the mechanism is not clear. Neutrophil extracellular traps (NETs) are a DNA skeleton coated with various proteases, and it is associated with various types of autoimmune nephritis. In this study, we examine whether NETs is involved in BPA-induced chronic kidney injury. In vivo, BPA exposure resulted in impaired renal function and altered renal morphology, including glomerular mesangial matrix expansion and increased renal interstitial fibroblast markers. Meanwhile, more dsDNA can be detected in the serum, and the NETs-associated proteins, MPO and citH3 were deposited in the renal system. In vitro, BPA and NETs treatment caused podocyte injury, a loss of marker proteins and disorder in the actin skeleton. After NETs inhibition via DNase administration, BPA-induced injuries were significantly relieved. In conclusion, the increase of NETosis in circulation and the renal system during BPA exposure suggests that NETs may be involved in BPA-induced chronic kidney injury.
\end{abstract}

\author{
Key Words \\ - bisphenol-A \\ - neutrophil extracellular \\ traps \\ - chronic kidney disease \\ - DNase
}

\section{Introduction}

Chronic kidney disease (CKD) is a group of kidney disorders characterized by a decline in the glomerular filtration rate with adverse outcomes, and it ultimately leads to end-stage renal disease (ESRD) (Levey et al. 2005). CKD is increasing worldwide and becoming one of the most serious global public health problems (Eckardt et al. 2013). CKD's clinical manifestations include but are not limited to edema, hypertension and proteinuria (Orth \& Ritz 1998, Ellison 2017). Understanding the pathological mechanism of CKD is important to provide strategies for its prevention and cure.

Bisphenol A is a widely used organic compound for industrial products, such as plastic bottles and food containers. In cases of high temperatures or incomplete polymerization, BPA could be released in food or drinking water and then absorbed into the body (Fenichel et al. 2013). Recently, there was more attention focused on whether exposure to BPA may lead to chronic diseases, 
such as heart disease, type 2 diabetes, obesity and chronic kidney disease (Rezg et al. 2014). According to the epidemiological investigations, there was a positive correlation between the levels of BPA and urinary albumin, and impaired renal function correlates with the increasing concentrations of plasma BPA (Krieter et al. 2013). However, the mechanism of pathology has not been thoroughly elucidated to date.

Neutrophil extracellular traps (NETs), which is also known as NETosis, is a DNA skeleton coated with cytosolic proteases, such as myeloperoxidase (MPO), citrullinated histone3 (citH3) and neutrophil elastase (NE). NETs is recognized as an innate immunity barrier that could capture and kill pathogens (Brinkmann et al. 2004), which is an important process during inflammation. However, excessive NETosis may cause physical dysfunction (Gardiner \& Andrews 2012, Nakazawa et al. 2017, Qi et al. 2017) and contribute to organ damage in some diseases such as atherosclerosis and rheumatoid arthritis (RS) (Warnatsch et al. 2015, Apel et al. 2018). CKD is accompanied by lowgrade chronic inflammation (Mihai et al. 2018), and NETosis can be detected in lupus erythematosus, acute kidney injury and ANCA (anti-neutrophil cytoplasmic antibodies)associated nephritis (Hakkim et al. 2010). However, there is no study on the role of NETs in the chronic kidney injuries induced by BPA. In this study, we explored whether NETs were involved in BPA-induced chronic kidney injury.

\section{Materials and methods}

\section{Animals}

Eight-week-old male C57BL/6 mice, which were purchased from Chongqing Medical University, were housed in specific pathogen-free conditions (SPF) and received a standard diet or a diet containing different amounts of BPA (5, 50 or $500 \mu \mathrm{g} / \mathrm{kg}$ per day) for 8 weeks. The different doses of BPA exposure were set according to methods used by the US Environmental Protection Agency and the European Food Safety Agency (Ma et al. 2018). Individual groups of mice were simultaneously treated with BPA at doses of $500 \mu \mathrm{g} / \mathrm{kg}$ per day and 200 U DNase twice a week via vein injection for 8 weeks. All procedures were approved by the Animal Care Committee of Chongqing Medical University. Urinary albumin (Abcam, ab108792), creatinine (cr, Bioassay, DICT-500) and blood urea nitrogen (BUN, Bioassay, DIUR-100) were measured by Elisa kits following the manufacturer's protocol.

\section{Cell culture and treatments}

Immortalized human podocytes were gifted by professor John C He from Nephrology Medicine at Mount Sinai. Podocytes were cultured in RPMI-1640 medium with 10\% FBS (Gibco, 10099141), 1\% PS (HyClone, SV30010) and 1\% ITS (Sigma, I-3146), and the cells were grown at $33^{\circ} \mathrm{C}$. After the cells reached $50 \%$ confluence, they were transferred to $37^{\circ} \mathrm{C}$ for 14 days to differentiate before any experimental manipulations. Podocytes were treated with BPA $(0.1 \mu \mathrm{mol} / \mathrm{L})$, NETs $(500 \mathrm{ng} / \mathrm{mL})$, DNase $(5 \mathrm{U} / \mathrm{mL})$ and BPA combined with NETs or D-NETs for $48 \mathrm{~h}$. D-NETs were incubated in $37^{\circ} \mathrm{C}$ for $1 \mathrm{~h}$ before use. The concentration of BPA was set as previously reported (Olea-Herrero et al. 2014), while the concentration of NETs was confirmed by an MTT test as previously reported (Warnatsch et al. 2015).

\section{MTT cell viability assay}

An MTT cell viability assay was used to confirm the NETs concentration in human podocyte cultures. Podocytes were seeded into 96-well flat bottom plates. After differentiating at $37^{\circ} \mathrm{C}$ for 14 days, the podocytes were treated with NETs at $100,300,500,700,1000 \mathrm{ng} / \mathrm{mL}$ for $48 \mathrm{~h}$. After removing the supernatant, medium without FBS and $10 \mu \mathrm{L}$ MTT work reagent (Amresco0793) were added to each well, and then incubated at $37^{\circ} \mathrm{C}$ for $4 \mathrm{~h}$ in the dark. Finally, $150 \mu \mathrm{L}$ DMSO were added to each well. After gently mixing the well contents, the optical density value (OD) was read in a Microplate Reader at $570 \mathrm{~nm}$.

\section{Immunohistochemical analysis}

For immunohistochemical analysis, kidney sections were dewaxed with xylene and hydrated using sequential ethanol (100, 95, 85 and 75\%) and distilled water washes. Antigen retrieval was performed by heating the sections in sodium citrate buffer, and then the sections were blocked with 3\% hydrogen peroxide and 5\% serum. Diluted primary antibodies were added and incubated overnight at $4^{\circ} \mathrm{C}$. The $\alpha$-SMA (Abcam, ab32575, 1:400) was used to examine renal interstitial fibrosis, MPO (Abcam, ab9535, 1:50) and citH3 (Abcam, ab5103, 1:200) represented NETs. To detect podocytes, Podocin (Abcam, ab50339, 1:400) and Nephrin (BOSTER, BA1669, 1:800) were used. After incubation with a secondary antibody, a peroxidase/ diaminobenzidine (DAB) detection system was used. Quantitation was assessed with ImageJ. Each group had at least nine randomly selected target areas from three different sections and was viewed by three independent investigators in a blinded manner. 


\section{Immunofluorescence staining}

Kidney frozen sections were fixed with methanol for $15 \mathrm{~min}$ at $-20^{\circ} \mathrm{C}$ and then blocked in blocking buffer for $60 \mathrm{~min}$. After aspirating the blocking solution, diluted primary antibodies were applied and incubated overnight at $4^{\circ} \mathrm{C}$. Podocin (Abcam, ab50339, 1:1000) and F-actin (Invitrogen, A34055, 1:50) were used to detect podocytes. The next day, sections were incubated with 488 (Green) or 555 (Red)-labeled secondary antibodies for $1 \mathrm{~h}$ at room temperature in the dark. For cell immunofluorescence, podocytes were cultured on 24-well plates containing glass slides, and then the above protocol was conducted.

\section{NETs isolation and image}

NETs were isolated as previously described (Warnatsch et al. 2015). Human neutrophils were extracted from healthy donors using Percoll as previously described (Brinkmann et al. 2010). Neutrophils were left in a 6-well plate or 24-well plate coated with glass slides and treated with BPA $(0.1 \mu \mathrm{mol} / \mathrm{L})$ or phorbol-12-myristate-13-acetate (PMA, 20 nmol) for $4 \mathrm{~h}$. PMA is a recognized drug that stimulates NETs production and was used as a positive control. Afterward, the culture medium was removed, and NETs were partially digested by the application of a restriction enzyme mix buffer combining the enzymes BseRI, PacI, NdeI and AfII (New England Biolabs) at a concentration of $5 \mathrm{U} / \mathrm{mL}$ in NEB buffer. The restriction digest was performed over $1 \mathrm{~h}$ at $37^{\circ} \mathrm{C}$ followed by centrifugation at $1000 \mathrm{~g}$ for $10 \mathrm{~min}$. The extracellular structure was confirmed with Sytox Green (Invitrogen, P11496), MPO and citH3.

\section{Quantification of NETs}

A Quant-iT Picogreen dsDNA Assay Kit (Invitrogen, P11496) was used to quantify neutrophil extracellular traps (NETs). Briefly, $100 \mu \mathrm{L}$ of standard or collected supernatant were transferred into separate black, opaque, flat-bottomed 96-well plates. Then, $100 \mu \mathrm{L}$ of Picogreen reagent were added to the samples after diluting the samples to 1:200 in TE buffer. After 10 min of incubation at room temperature in the dark, extracellular DNA was quantified using a standard curve based on known concentrations of DNA. Fluorescence values were recorded on a Fluorescent Microplate Reader using excitation/ emission settings of 485 and $520 \mathrm{~nm}$.

\section{Quantification of serum myeloperoxidase}

Mice serum MPO content was detected with an Elisa kit (Thermo, Mouse Myeloperoxidase ELISA Kit). All reagents and samples were brought to room temperature before use, and then $100 \mu \mathrm{L}$ of each standard and sample were added to the appropriate wells and incubated for $2.5 \mathrm{~h}$ at room temperature. The solution was discarded and washed four times with Wash Buffer. Then, $100 \mu \mathrm{L}$ of prepared biotinylated antibody (diluted 80 -fold with Diluent Buffer) were added to each well. The samples were incubated for $1 \mathrm{~h}$ at room temperature with gentle shaking. After washing four times with Wash Buffer, the solution was discarded, and the $100 \mu \mathrm{L}$ of prepared Streptavidin-HRP solution (diluted 400-fold with Diluent Buffer) were added to each well. The samples were incubated for $45 \mathrm{~min}$ at room temperature with gentle shaking. Next, after washing four times with Wash Buffer, $100 \mu \mathrm{L}$ of TMB Substrate were added to each well. The samples were incubated for $30 \mathrm{~min}$ at room temperature in the dark with gentle shaking, and then measurements were recorded on a Microplate Reader at $450 \mathrm{~nm}$.

\section{Western blot analysis}

The total proteins from cultured podocytes were collected and the protein concentrations were determined with a BCA assay kit (P0010S; Beyotime, Shanghai, China). The total cell proteins $(50 \mu \mathrm{g})$ from each sample were separated by SDS-PAGE and transferred to a PVDF membrane. After blocking the membrane with $5 \%$ skim milk for $1 \mathrm{~h}$, the membranes were incubated with Podocin (ab50339, 1:1000; Abcam), synaptopodin (sc-515842, 1:250; Santa Cruz), Nephrin (BA1669, 1:250; Boster, Wuhan, China) and $\beta$-actin (BM5422, 1:400; Boster) at $4^{\circ} \mathrm{C}$ overnight, after washing three times with Tris-buffered saline solution/ Tween (TBST), and then the membrane was incubated with the appropriate secondary antibody (CST). The grayscale value of each protein was standardized with $\beta$-actin and analyzed by Fusion.

\section{Transmission electron microscopy}

Electron microscopic sample handling and detection were performed by the electron microscope lab of Chongqing Medical University. Kidney tissue from each group of mice was prepared as described previously (Liu et al. 2018). Glomeruli were randomly selected from each mouse. Mouse kidneys with appropriate sizes were fixed in $2.5 \%$ glutaraldehyde for $24 \mathrm{~h}$ at $4^{\circ} \mathrm{C}$, and then 
they were post-fixed in $1 \%$ osmium tetroxide and $0.1 \%$ potassium ferricyanide. After washing the tissue with $0.1 \mathrm{M}$ cacodylate buffer, the samples were dehydrated through a graded series of ethanol (30-90\%) washes and embedded in a mixture of epoxy resin.

\section{Statistics}

SPSS version 17 (SPSS Inc) was used for statistical analysis. All the values that were reported are presented as the means \pm standard error of means (s.E.M.). Differences between groups were analyzed with one-way ANOVA or nonparametric method (multiple comparisons for normal distribution or abnormal distribution, respectively). $P<0.05$ was considered to be statistically significant.

\section{Result}

\section{BPA-induced kidney injury and impaired renal function in C57BL/6 mice}

First, we determined whether BPA exposure induced kidney injuries in C57BL/6 mice as described in other rodent models. After 8 weeks of BPA exposure, a significant increase in serum creatinine and blood urea nitrogen (BUN) occurred in a dose-dependent manner (Fig. 1A and B). Both serum creatinine and BUN were significantly increased for the 50 and $500 \mu \mathrm{g} / \mathrm{kg} /$ day BPA exposure doses. Furthermore, kidney histology showed that the glomerular mesangial matrix area was expanded in mice exposed to BPA at 50 or $500 \mu \mathrm{g} / \mathrm{kg} /$ day compared to control mice, and no obvious changes were observed in mice exposed to BPA at $5 \mu \mathrm{g} / \mathrm{kg} /$ day (Fig. 1C). Apparently renal interstitial fibrosis was exhibited by Sirius Red staining after exposure to BPA at $500 \mu \mathrm{g} / \mathrm{kg} /$ day (Fig. 1D). Meanwhile, $\alpha$-SMA, which is the fibroblast marker, was increased substantially after exposure to different doses of BPA (Fig. 1D). These data showed that BPA exposure could result in a significant kidney injury in C57BL/6 mice, including impaired renal function and changes in renal morphology.

\section{NETs formation was increased in BPA-exposed C57BL/6 mice}

Our experiments have showed that BPA could induce kidney injuries in C57BL/6 mice. To explore the role of NETs in the kidney injury induced by BPA, dsDNA,

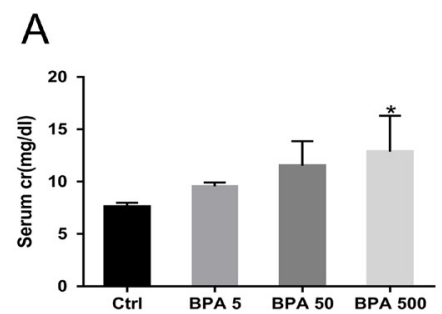

C

BPA 5

BPA 50
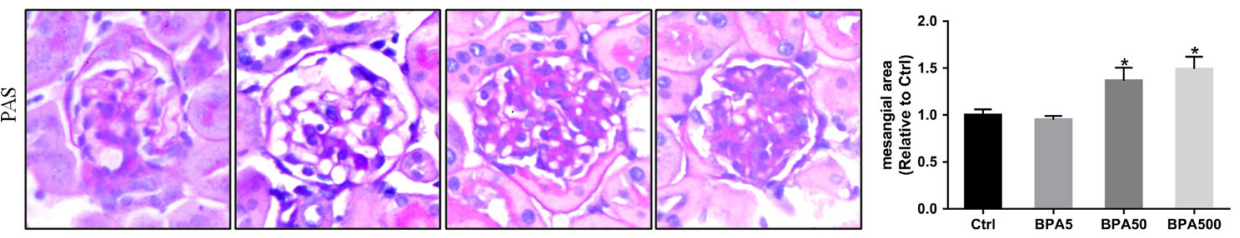

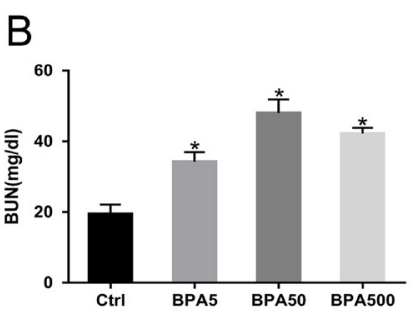

D

Ctrl

BPA 5

BPA 50

BPA 500
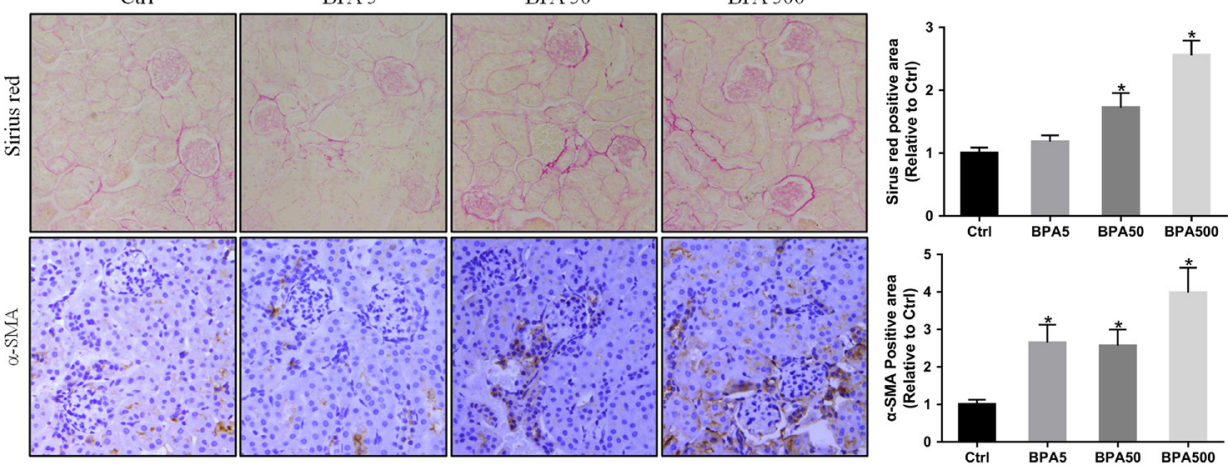

Figure 1

BPA induces kidney injury and decline in renal function in C57BL/6 mice. Concentrations of serum creatinine (A) and blood urea nitrogen (B) in C57BL/6 mice after exposed to BPA at 5, 50 or $500 \mu \mathrm{g} / \mathrm{kg}$ per day for 8 weeks ( $n=5 /$ group). (C) Periodic Acid-Schiff (PAS) staining and quantifications for the mesangial matrix area in different groups. (D) Representative image and quantifications of Sirius Red staining and $\alpha$-SMA immunostaining for interstitial fibrosis detection. One-way ANOVA was used for statistical analysis, $* P<0.05$ vs control group, numeric data are presented as means \pm S.E.M. A full colour version of this figure is available at https://doi.org/10.1530/JOE-18-0608. 
which is the basic structure of NETs, was detected first. Compared to the control group, serum dsDNA was increased after BPA exposure at 50 or $500 \mu \mathrm{g} / \mathrm{kg} / \mathrm{day}$. Moreover, MPO, which is the coated protease of NETs, was also increased in serum from mice exposed to BPA at 50 or $500 \mu \mathrm{g} / \mathrm{kg} /$ day compared to the control mice, which is the same tendency observed for dsDNA (Fig. 2A and B). Immunohistochemistry demonstrated that both citH3 and MPO, which are NETs-associated markers, were deposited in the renal system in a dose-dependent manner, especially in the glomerular area and for the mice exposed to BPA at $500 \mu \mathrm{g} / \mathrm{kg} /$ day (Fig. 2C). To verify whether BPA could induce NETosis in vitro, neutrophils from human blood were extracted and cultured with BPA. According to the experiments in vivo, the dsDNA level in the supernatant of cultured neutrophils was significantly increased after BPA treatment (Fig. 2D). In addition, both thread-like DNA structures identified by SYTOX green and enhanced MPO and citH3 expression verified by immunofluorescence suggested there was an increase in the release of NETs after neutrophils were cultured with BPA (Fig. 2E).

\section{DNasel relieved BPA-induced kidney injuries}

According to the in vivo and in vitro experiments, increased NETosis was found after BPA exposure. Then, we wondered whether suppressed NETosis could relieve BPA-induced kidney injury. Considering the obvious kidney injury and NETs deposition after BPA exposure, mice treated with BPA at a dose of $500 \mu \mathrm{g} / \mathrm{kg}$ were simultaneously treated with DNase, which could inhibit NETosis by degrading the DNA structure. After DNase injection, serum dsDNA and MPO decreased significantly compared with single BPA exposure (Fig. 3A and B). Reduced expression of NETs-related protein citH3 and MPO confirmed NETosis was inhibited in the kidneys (Fig. 3C). Proteinuria is a feature of chronic kidney injury, and it mainly occurs due
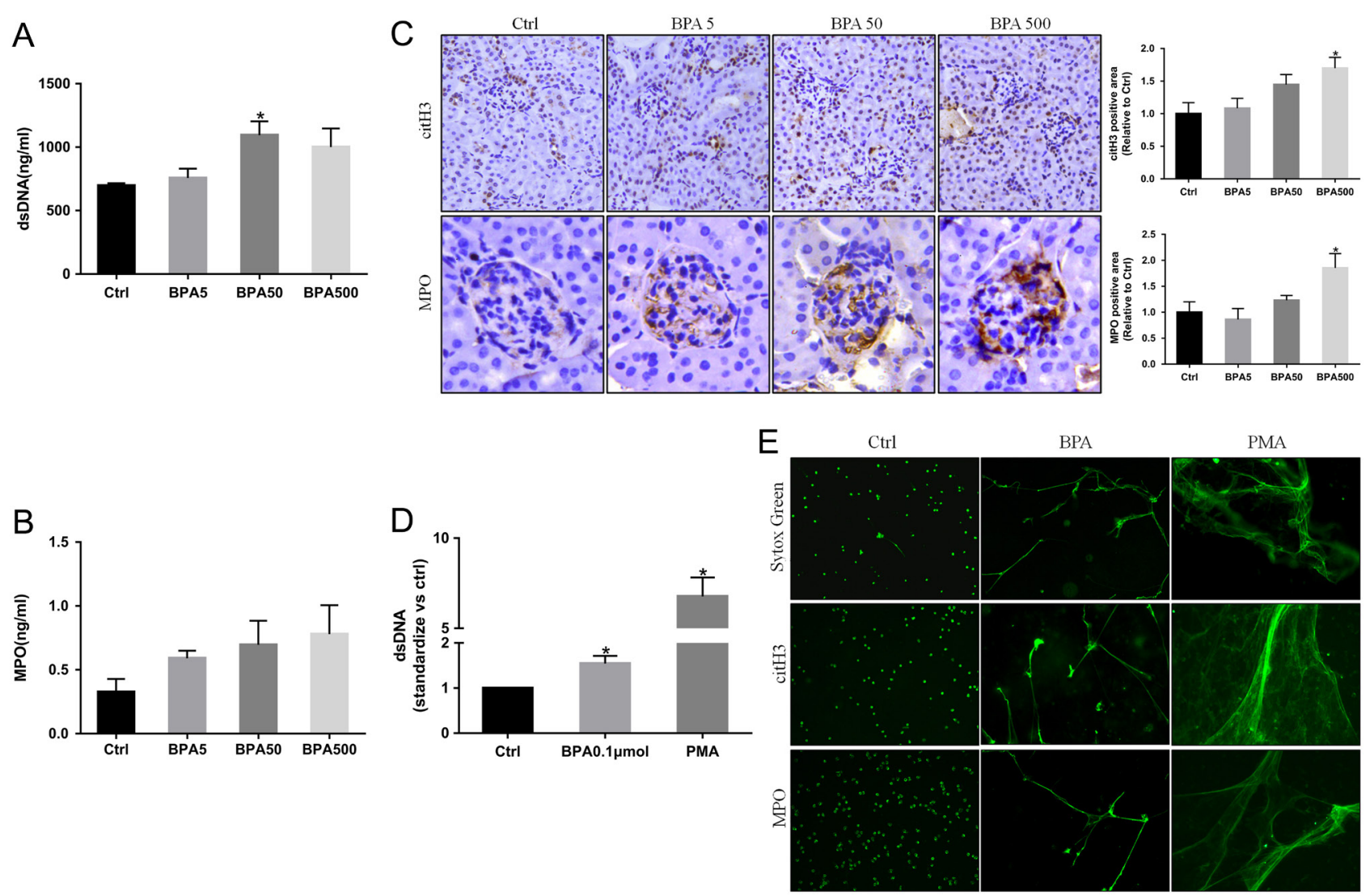

Figure 2

BPA upregulates NETs associate protein expression. Concentrations of serum dsDNA (A) and MPO (B) in C57BL/6 mice after exposed to different BPA levels (C). Immunostaining of NETs-related marker MPO and citH3 in mice kidney (D). Concentrations of dsDNA in cultured neutrophil supernatant after different treatments, PMAs were set as a positive control ( $n=7 /$ group) (E). Immunofluorescent staining for NETs-related marker dsDNA, citH3 and MPO in neutrophils. One-way ANOVA was used for statistical analysis in (A), (B), (C), nonparametric method was used for statistical analysis in (D), *P $<0.05$ vs control group, numeric data are presented as means \pm S.E.M. 
to an impaired glomerular filtration barrier. BPA exposure could result in increased urinary albumin excretion, and the increased proteinuria was controlled after NETosis was inhibited by DNase (Fig. 4A). Decreased BUN also indicated there was improved renal function after DNase injection (Fig. 4B). Along with this result, a reduced glomerulus mesangial matrix area and decreased collagen deposition also showed alleviated glomerulosclerosis and renal interstitial nephritis after DNase injection (Fig. 4C and D). These data further confirmed the role of NETosis in BPA-induced kidney injury.

\section{BPA induced podocytopathy via increased NETosis both in vivo and in vitro}

Podocytes, which are terminally differentiated epithelial cells, are the inner layer of the glomerular filtration barrier. Proteinuria is attributed to the damage or dysfunction of podocytes, especially changes in slit diaphragmassociated proteins, such as nephrin, podocin and CD2AP. BPA exposure led to increased urinary albumin excretion in C57BL/6 mice, which indicates there were potential podocyte injuries. In vivo, we observed distinct foot process effacement and irregular glomerular basement membrane thickening in the mice after exposure to BPA at $500 \mu \mathrm{g} / \mathrm{kg} / \mathrm{day}$ using a transmission electron microscope (TEM) (Fig. 5A). The expression of the podocyte differentiation markers podocin and nephrin was significantly reduced after BPA exposure (Fig. 5B and C), which implied BPA exposure was a harm for the podocytes. After NETs were inhibited by Dnase, a significant protective effect for podocytes was found, which was presented as the ordered arrangement of the foot process and regular expression of the slit diaphragmassociated proteins podocin and nephrin (Fig. 5A, B and C). In vitro, NETs stimulated by BPA were collected and used to treat podocytes. Compared to the control or single BPA treatments, podocyte injuries were more serious after treatment with NETs or NETs combined with BPA, which was confirmed by decreased protein expression of podocin and nephrin (Fig. 5D and E). Podocytes treated with NETs or NETs combined with BPA also exhibited obvious disordered actin structures and less microfilament (Fig. 5F and G). DNase was used in NETs-treated podocytes, which are described as D-NETs. As we observed in the rodent model, pretreatment with DNase significantly ameliorated
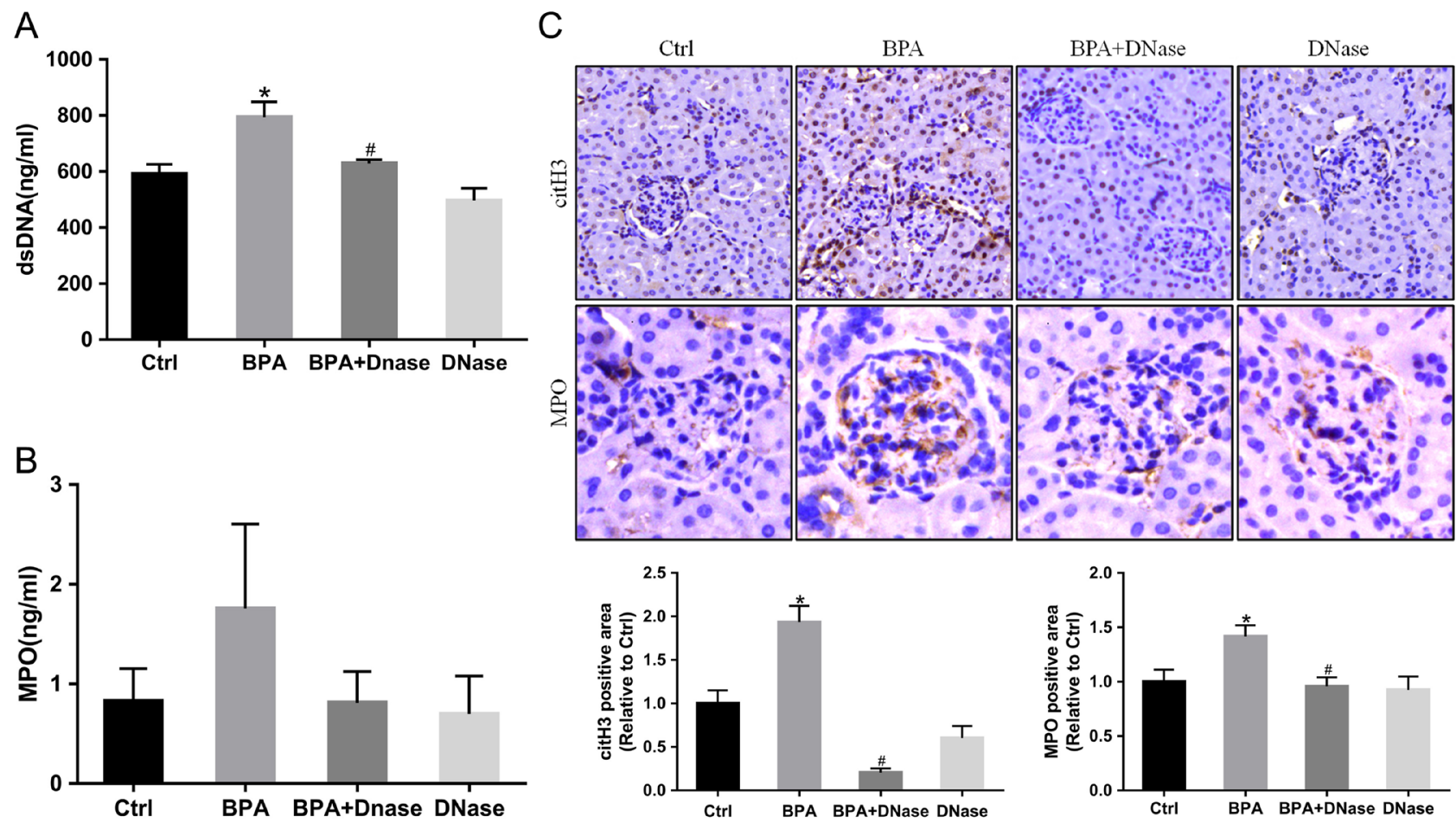

Figure 3

DNase injection reduces NETs formation in C57BL/6 mice. Concentrations of serum dsDNA (A) and MPO (B) in C57BL/6 mice after exposure to BPA at $500 \mu \mathrm{g} / \mathrm{kg}$ per day and DNase injection for 8 weeks ( $n=5,8,5,3$ for Ctrl, BPA500, BPA + DNase, DNase, respectively). (C) Representative image and quantifications of NETs-related markers MPO and citH3 in mice kidney. One-way ANOVA was used for statistical analysis, $* P<0.05$ vs control group, $\# P<0.05$ vs BPA group; numeric data are presented as means \pm S.E.M. A full colour version of this figure is available at https://doi.org/10.1530/JOE-18-0608. 

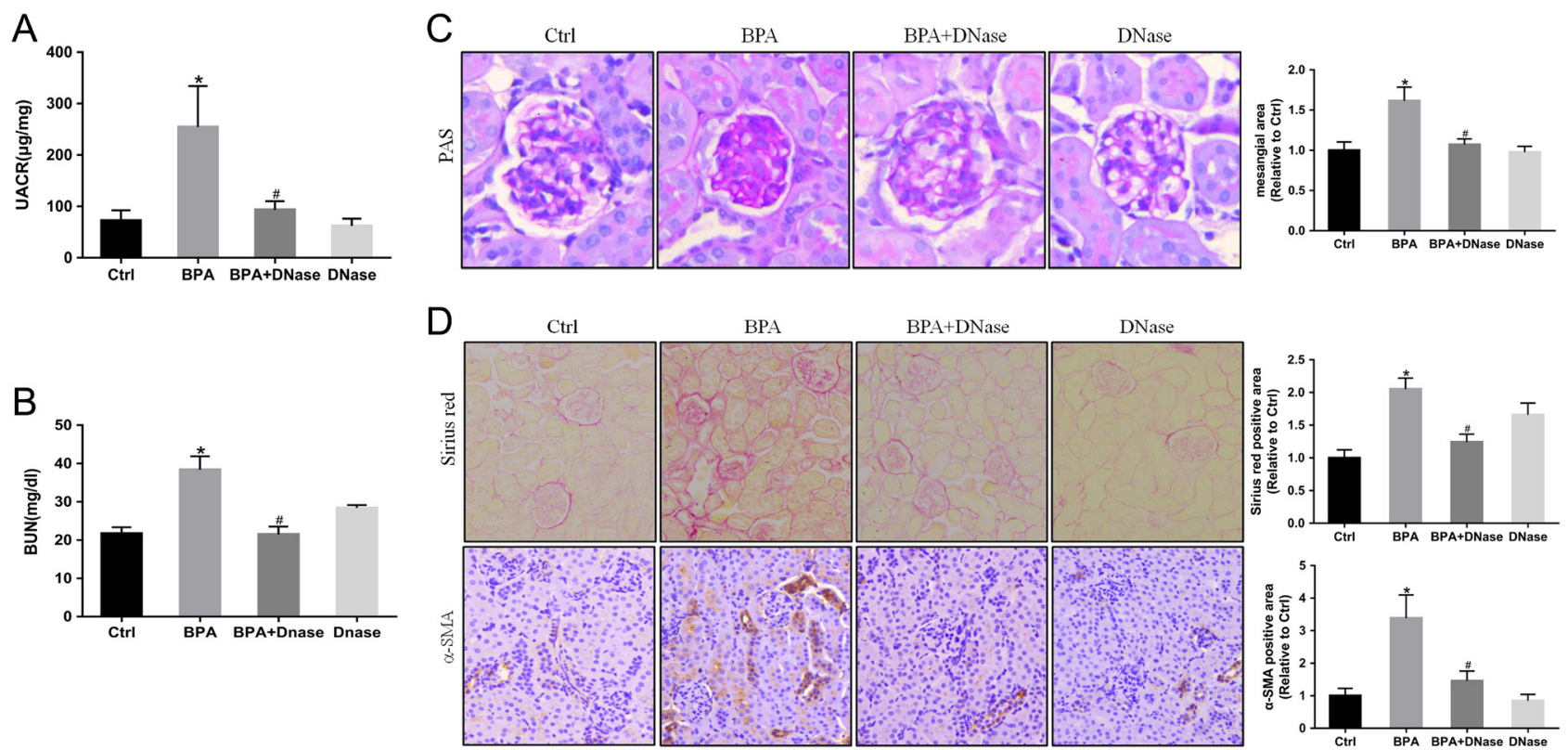

Figure 4

DNase injection relieves BPA-induced kidney injury in C57BL/6 mice. (A) UACR (urine albumin-to-creatinine ratio) in C57BL/6 mice after exposure to BPA or DNase injection. (B) Concentration of blood urea nitrogen in C57BL/6 mice after exposure to BPA or DNase injection. (C) Periodic Acid-Schiff (PAS) staining and quantifications for the mesangial matrix area in different groups. (D) Representative image and quantifications of Sirius Red staining and $\alpha$-SMA immunostaining for interstitial fibrosis. One-way ANOVA was used for statistical analysis, $* P<0.05$ vs control group, \#P<0.05 vs BPA group, numeric data are presented as means \pm S.E.M. A full colour version of this figure is available at https://doi.org/10.1530/JOE-18-0608.

podocyte injuries, including recovering the nephrin and podocin expression as well as increasing F-actin (Fig. 5D, $\mathrm{E}, \mathrm{F}$ and $\mathrm{G})$. These results suggest that increased NETosis induced by BPA could result in podocyte injuries.

\section{Discussion}

Our study confirmed that NETs play an important role in BPA-induced chronic kidney injury. The presence of NETs could be detected in blood and kidneys after BPA exposure in C57BL/6 mice, especially in the glomerulus, which may explain the increased urinary albumin and decreased creatinine clearance. A comprehensive amelioration of kidney injuries confirms the effect of DNase, which provides a new perspective that may benefit current knowledge of BPA exposure.

As one of the most highly produced toxic chemicals, BPA exposure could occur in humans through a variety of ways (Rezg et al. 2014). Part of intracorporal BPA could be eliminated through the kidney, but there could still be some remaining BPA that could be detected in body fluids and may cause chronic damage, such as cardiovascular disease, diabetes, hypertension and chronic kidney disease (Fenichel et al. 2013,
Krieter et al. 2013, Rezg et al. 2014). Trasande's study found that urinary BPA levels are associated with microalbuminuria in American children (Trasande et al. 2013), and a subsequent study reached the same conclusion for American adolescents (Trasande et al. 2014). In 2012, the team of Professor Ning conducted a survey for the first time in the Chinese population. The results showed that $87.5 \%$ of people over 40 years old in Shanghai had detectable BPA in their urine and had a positive relationship with low proteinuria ( $\mathrm{Li}$ et al. 2012). In our previous study, it was confirmed that serum BPA levels were positively correlated with the risk of developing CKD in patients with type 2 diabetes or hypertension ( $\mathrm{Hu}$ et al. 2015, 2016). In rodents, rats exposed to BPA showed renal damage, which was demonstrated by decreased creatinine clearance, the presence of proteinuria and glomerulosclerosis (Kobroob et al. 2018). CD1 mice have been observed to develop proteinuria after BPA intraperitoneal injection accompanied by damage to the glomerular filtration barrier and an increasing glomerular mesangial matrix area (Olea-Herrero et al. 2014), which was the same outcome we observed for C57BL/6 mice. Moreover, significant renal interstitial fibrosis has been confirmed. These research studies corroborate the relationship 


\begin{tabular}{l|l|l|r|r|}
$\begin{array}{l}\text { Journal of } \\
\text { Endocrinology }\end{array}$ & S Tong et al. & $\begin{array}{l}\text { Bisphenol-A and chronic } \\
\text { kidney disease }\end{array}$ & $\mathbf{2 4 1 : 2}$ & 132 \\
\hline
\end{tabular}

A

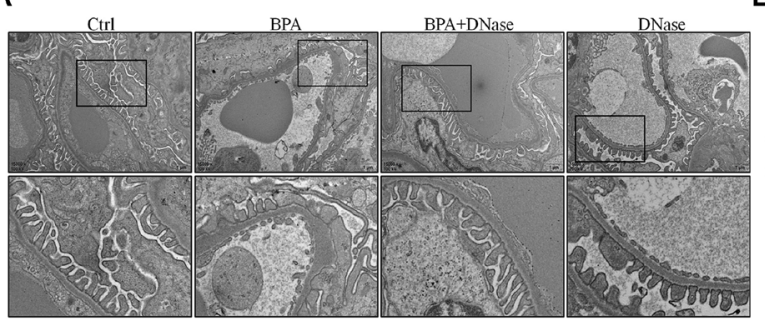

B
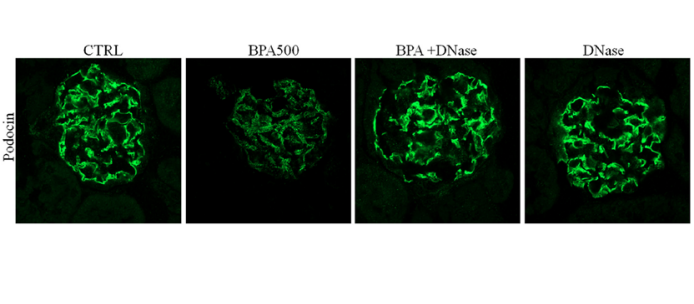

C
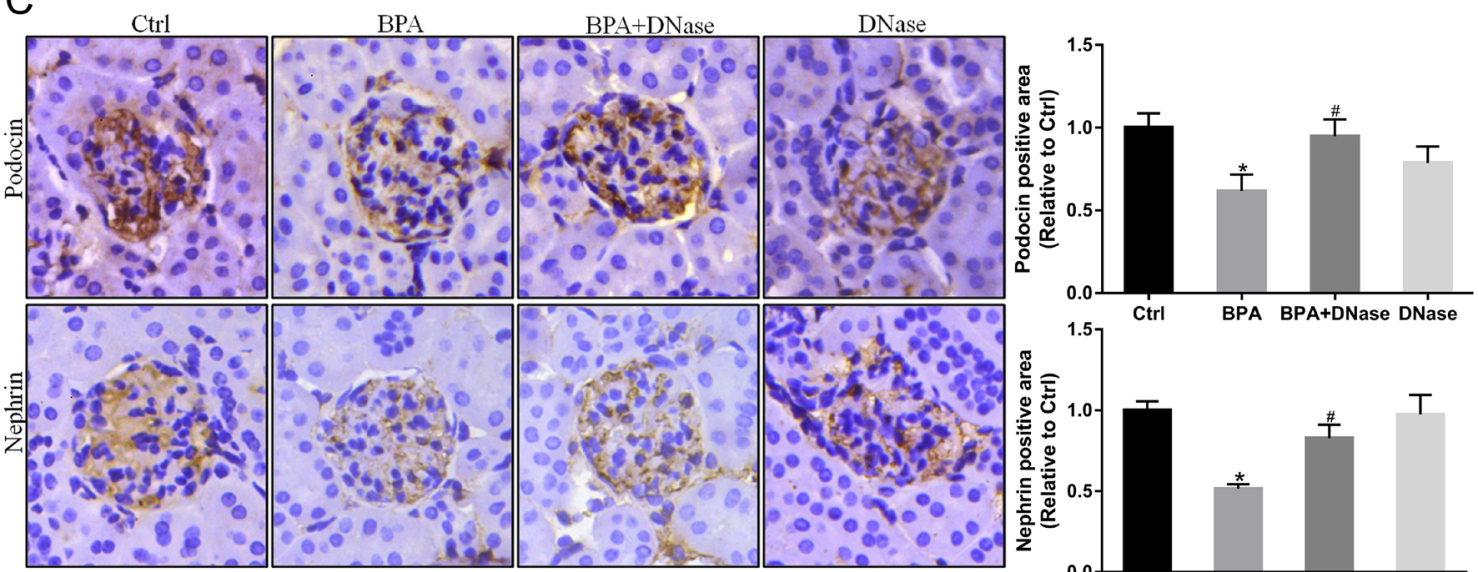

D

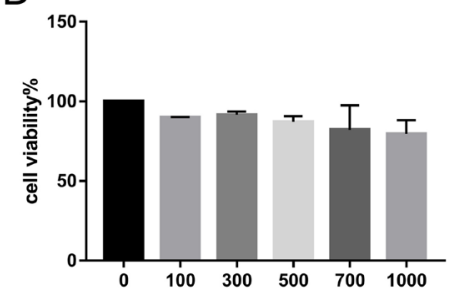

E

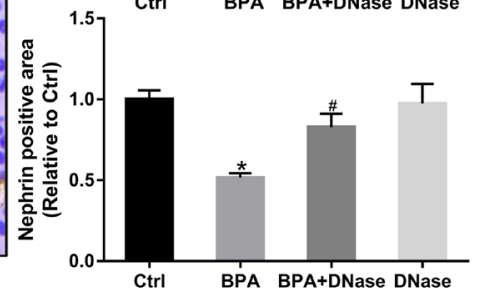

F
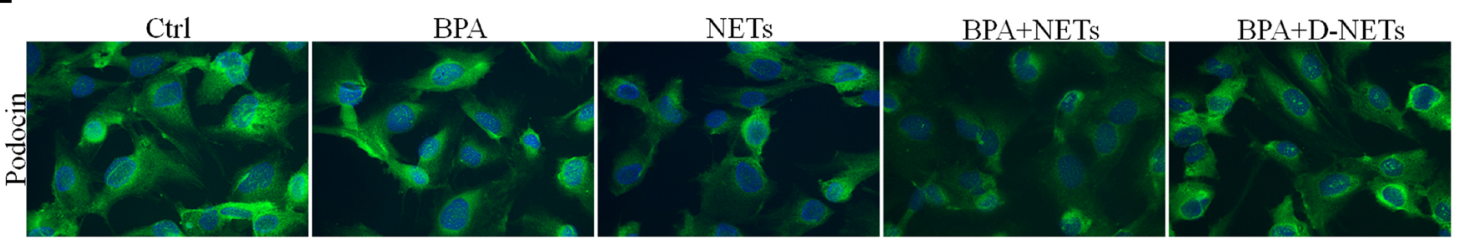

G

Ctrl
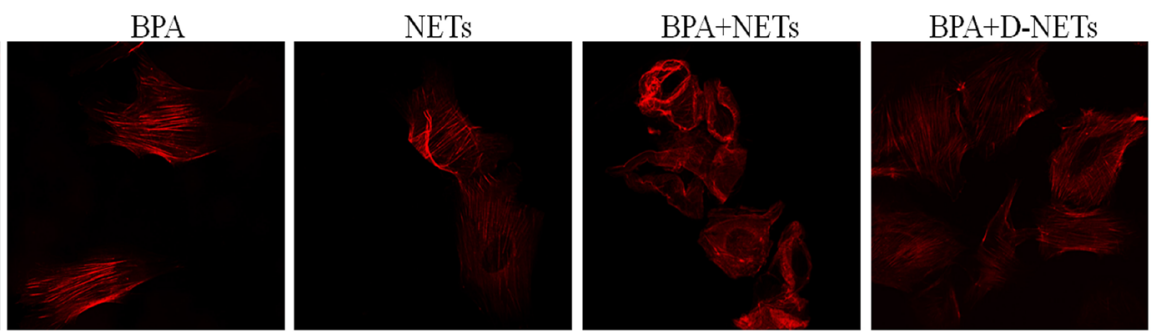

Figure 5

BPA exposure lead to destructed GBM and decreased podocyte marker. (A) Representative photomicrographs of glomerular basement membrane (GBM) image by transmission electron microscopy (TEM). (B) Immunofluorescent staining of podocin in glomerular of C57BL/6 mice in different groups. (C) Representative image and quantifications of podocyte marker podocin and nephrin. (D) MTT test for appropriate concentration of NETs to treat podocyte. (E) Protein expression of podocyte marker in podocyte after BPA $(0.01 \mu \mathrm{mol} / \mathrm{L})$ with (or) NETs $(500 \mathrm{ng} / \mathrm{mL})$ treatment for $48 \mathrm{~h}$.

Immunofluorescent staining of podocin (F) and F-actin (G) in podocyte. One-way ANOVA was used for statistical analysis, $* P<0.05$ vs control group, $\# P<0.05$ vs BPA group, \& $P<0.05$ vs BPA + NETs group; numeric data are presented as means \pm S.E.M. 
between BPA and chronic kidney injury. Although this conclusion is clear, there is no universal conclusion on what the specific injury mechanism is.

NETs were first described in 2004. After stimulation by a pathogen or infection, neutrophils undergo chromatin depolymerization, membrane rupture and finally release intracellular chromatin DNA and granule proteins into the extracellular space to form a network structure that is described as NETosis. NETs could encapsulate pathogens effectively (Jorch \& Kubes 2017). However, excessive formation or abnormal elimination of NETs may be involved in the development of inflammation and various metabolic diseases, including some kidney diseases and especially immune-related kidney disease (Lee et al. 2017). Considering that inflammation is an important part of the process of CKD, we hypothesize that NETs may be involved in BPA-induced chronic kidney injury.

To confirm this hypothesis, we detected NETs-related markers in serum and kidney tissue. dsDNA, MPO and citH3 were increased after BPA exposure, especially in the glomerular area, which suggests that there is increasing NETosis in the glomerulus. Podocytes were significantly damaged, as well. Moreover, we observed relieved renal function, restored morphology and improved podocyte function after NETosis was inhibited by DNase, which confirmed the role of NETs in the process of BPA-induced kidney injury. DNase has also been reported in other NETsrelated kidney injuries. Seredkina et al. (2009) and Napirei et al. (2006) found that the decline of DNase1 activity in the kidneys could influence chromatin disposal and may be responsible for transformation of mild mesangial lupus nephritis into ESRD. For the source of NETosis, as the most abundant leukocyte in the blood, neutrophils were present in ordinary kidneys or under inflammation conditions. BPA-stimulated NETosis may occur both in circulation and kidney tissue. We observed damaged glomerular filtration barriers under TEM, and released NETs from circulation may enter the glomerulus through the damaged glomerular basement membrane. Subsequent research about renal endothelial cells may confirm this hypothesis.

As an endocrine disruptor, BPA can participate in various signaling pathways in vivo. Hui found that lowdose BPA can increase the migration and invasion of SKOV3 and A2780 cells, and it can induce epithelialmesenchymal transition through the Wnt signaling pathway (Hui et al. 2018). Podocytes are one of the intrinsic cells of the glomerulus, and as the terminally differentiated epithelial cells, they consist of three parts: the cell body, major process and foot process (Smoyer \& Mundel 1998). A slit diaphragm is formed by adjacent foot processes, and it is a critical structure for preventing proteinuria (Welsh \& Saleem 2011). Podocyte injury and the following protein leakage were typical symptoms and an important link of CKD, which also accelerated the sclerosis of the glomerulus. Previous studies have noted that BPA exposure leads to podocyte hypertrophy, and both TGF- $\beta 1$ and collagen IV were upregulated after BPA stimulation, which indicates glomerulosclerosis and potential renal interstitial fibrosis (Olea-Herrero et al. 2014). As expected, podocyte differentiation markers were significantly decreased after BPA exposure, which represents the destruction of podocyte actin and is consistent with increased urinary albumin in mice. In vitro, we found that BPA or BPA-stimulated NETs obviously induce podocyte injury, while the injuries caused by BPA combined with NETs in podocytes were the most serious, which indicates that BPA causes direct damage to podocytes, but the damage caused by stimulated NETosis is more substantial.

Taken together, our study suggests that exposure to BPA can lead to chronic kidney injury, including damage to the glomerular filtration barrier and occurrence of proteinuria. Our study first showed that BPA-induced CKD was accompanied by increased NETosis. Due to the poor prognosis of $\mathrm{CKD}$, we found the key to delay its progression is very important. BPA is one of the risk factors for chronic kidney disease, and DNase could mitigate BPAinduced kidney injury, which may represent a target for preventing and treating chronic kidney disease.

\section{Declaration of interest}

The authors declare that there is no conflict of interest that could be perceived as prejudicing the impartiality of the research reported.

\section{Funding}

This research was funded by the National Natural Science Foundation of China (grant number 81670785, 81602821, 81800731, 81870567, 81700754, 81770851), Merck Serono Diabetes Research Found, and the Fundamental Science \& Advanced Technology Research of Chongqing (Major Project, grant number cstc2015jcyjBX0096), National Key Research \& Development Plan, Major Project of Precision Medicine Research (2017YFC0909600, subproject: 2017YFC090960202017YFC0909603). Thanks to the Chongqing Key Laboratory of Translational Medicine in Major Metabolic Diseases.

\section{References}

Apel F, Zychlinsky A \& Kenny EF 2018 The role of neutrophil extracellular traps in rheumatic diseases. Nature Reviews Rheumatology 14 467-475. (https://doi.org/10.1038/s41584-018-0039-z)

Brinkmann V, Laube B, Abu Abed U, Goosmann C \& Zychlinsky A 2010 Neutrophil extracellular traps: how to generate and visualize them. Journal of Visualized Experiments 36 e1724. (https://doi. $\operatorname{org} / 10.3791 / 1724)$ 
Brinkmann V, Reichard U, Goosmann C, Fauler B, Uhlemann Y, Weiss DS, Weinrauch Y \& Zychlinsky A 2004 Neutrophil extracellular traps kill bacteria. Science 303 1532-1535. (https://doi.org/10.1126/ science.1092385)

Eckardt KU, Coresh J, Devuyst O, Johnson RJ, Kottgen A, Levey AS \& Levin A 2013 Evolving importance of kidney disease: from subspecialty to global health burden. Lancet 382 158-169. (https:// doi.org/10.1016/S0140-6736(13)60439-0)

Ellison DH 2017 Treatment of disorders of sodium balance in chronic kidney disease. Advances in Chronic Kidney Disease 24 332-341. (https://doi.org/10.1053/j.ackd.2017.07.003)

Fenichel P, Chevalier N \& Brucker-Davis F 2013 Bisphenol A: an endocrine and metabolic disruptor. Annales d'Endocrinologie $\mathbf{7 4}$ 211-220. (https://doi.org/10.1016/j.ando.2013.04.002)

Gardiner EE \& Andrews RK 2012 Neutrophil extracellular traps (NETs) and infection-related vascular dysfunction. Blood Reviews 26 255-259. (https://doi.org/10.1016/j.blre.2012.09.001)

Hakkim A, Furnrohr BG, Amann K, Laube B, Abed UA, Brinkmann V, Herrmann M, Voll RE \& Zychlinsky A 2010 Impairment of neutrophil extracellular trap degradation is associated with lupus nephritis. PNAS 107 9813-9818. (https://doi.org/10.1073/pnas.0909927107)

Hu J, Wang Y, Xiang X, Peng C, Gao R, Goswami R, Zhou H, Zhang Y, Zhen Q, Cheng Q, et al. 2016 Serum bisphenol A as a predictor of chronic kidney disease progression in primary hypertension: a 6-year prospective study. Journal of Hypertension 34 332-337. (https://doi. org/10.1097/HJH.0000000000000780)

Hu J, Yang S, Wang Y, Goswami R, Peng C, Gao R, Zhou H, Zhang Y, Cheng Q, Zhen Q et al. 2015 Serum bisphenol A and progression of type 2 diabetic nephropathy: a 6-year prospective study. Acta Diabetologica 52 1135-1141. (https://doi.org/10.1007/s00592-015-0801-5)

Hui L, Li H, Lu G, Chen Z, Sun W, Shi Y, Fu Z, Huang B, Zhu X, Lu W, et al. 2018 Low dose of bisphenol A modulates ovarian cancer gene expression profile and promotes epithelial to mesenchymal transition via canonical Wnt pathway. Toxicological Sciences 164 527-538. (https://doi.org/10.1093/toxsci/kfy107)

Jorch SK \& Kubes P 2017 An emerging role for neutrophil extracellular traps in noninfectious disease. Nature Medicine 23 279-287. (https:// doi.org/10.1038/nm.4294)

Kobroob A, Peerapanyasut W, Chattipakorn N \& Wongmekiat O 2018 Damaging effects of bisphenol A on the kidney and the protection by melatonin: emerging evidences from in vivo and in vitro studies. Oxidative Medicine and Cellular Longevity 2018 3082438. (https://doi. org $/ 10.1155 / 2018 / 3082438)$

Krieter DH, Canaud B, Lemke HD, Rodriguez A, Morgenroth A, von Appen K, Dragoun GP \& Wanner C 2013 Bisphenol A in chronic kidney disease. Artificial Organs 37 283-290. (https://doi.org/10.1111/ j.1525-1594.2012.01556.x)

Lee KH, Kronbichler A, Park DD, Park Y, Moon H, Kim H, Choi JH, Choi Y, Shim S, Lyu IS, et al. 2017 Neutrophil extracellular traps (NETs) in autoimmune diseases: a comprehensive review. Autoimmunity Reviews 16 1160-1173. (https://doi.org/10.1016/j.autrev.2017.09.012)

Levey AS, Eckardt KU, Tsukamoto Y, Levin A, Coresh J, Rossert J, De Zeeuw D, Hostetter TH, Lameire N \& Eknoyan G 2005 Definition and classification of chronic kidney disease: a position statement from Kidney Disease: Improving Global Outcomes (KDIGO). Kidney International 672089 2100. (https://doi.org/10.1111/j.1523-1755.2005.00365.x)

Li M, Bi Y, Qi L, Wang T, Xu M, Huang Y, Xu Y, Chen Y, Lu J, Wang W, et al. 2012 Exposure to bisphenol $\mathrm{A}$ is associated with low-grade albuminuria in Chinese adults. Kidney International 81 1131-1139. (https://doi.org/10.1038/ki.2012.6)
Liu T, Chen XM, Sun JY, Jiang XS, Wu Y, Yang S, Huang HZ, Ruan XZ \& Du XG 2018 Palmitic acid-induced podocyte apoptosis via the reactive oxygen species-dependent mitochondrial pathway. Kidney and Blood Pressure Research 43 206-219. (https://doi. org/10.1159/000487673)

Ma L, Hu J, Li J, Yang Y, Zhang L, Zou L, Gao R, Peng C, Wang Y, Luo T, et al. 2018 Bisphenol A promotes hyperuricemia via activating xanthine oxidase. FASEB Journal 32 1007-1016. (https://doi. org/10.1096/fj.201700755R)

Mihai S, Codrici E, Popescu ID, Enciu AM, Albulescu L, Necula LG, Mambet C, Anton G \& Tanase C 2018 Inflammation-related mechanisms in chronic kidney disease prediction, progression, and outcome. Journal of Immunology Research 2018 2180373. (https://doi. org/10.1155/2018/2180373)

Nakazawa D, Kumar SV, Marschner J, Desai J, Holderied A, Rath L, Kraft F, Lei Y, Fukasawa Y, Moeckel GW, et al. 2017 Histones and neutrophil extracellular traps enhance tubular necrosis and remote organ injury in ischemic AKI. Journal of the American Society of Nephrology $\mathbf{2 8}$ 1753-1768. (https://doi.org/10.1681/ASN.2016080925)

Napirei M, Gultekin A, Kloeckl T, Moroy T, Frostegard J \& Mannherz HG 2006 Systemic lupus-erythematosus: deoxyribonuclease 1 in necrotic chromatin disposal. International Journal of Biochemistry and Cell Biology 38 297-306. (https://doi.org/10.1016/j.biocel.2005.10.023)

Olea-Herrero N, Arenas MI, Munoz-Moreno C, Moreno-GómezToledano R, González-Santander M, Arribas I, Bosch RJ \& GonzalezSantander M 2014 Bisphenol-A induces podocytopathy with proteinuria in mice. Journal of Cellular Physiology 229 2057-2066. (https://doi.org/10.1002/jcp.24665)

Orth SR \& Ritz E 1998 The nephrotic syndrome. New England Journal of Medicine 338 1202-1211. (https://doi.org/10.1056/ NEJM199804233381707)

Qi H, Yang S \& Zhang L 2017 Neutrophil extracellular traps and endothelial dysfunction in atherosclerosis and thrombosis. Frontiers in Immunology 8 928. (https://doi.org/10.3389/fimmu.2017.00928)

Rezg R, El-Fazaa S, Gharbi N \& Mornagui B 2014 Bisphenol A and human chronic diseases: current evidences, possible mechanisms, and future perspectives. Environment International 64 83-90. (https://doi. org/10.1016/j.envint.2013.12.007)

Seredkina N, Zykova SN \& Rekvig OP 2009 Progression of murine lupus nephritis is linked to acquired renal Dnase1 deficiency and not to up-regulated apoptosis. American Journal of Pathology 175 97-106. (https://doi.org/10.2353/ajpath.2009.080943)

Smoyer WE \& Mundel P 1998 Regulation of podocyte structure during the development of nephrotic syndrome. Journal of Molecular Medicine 76 172-183. (https://doi.org/10.1007/s001090050206)

Trasande L, Attina TM \& Trachtman H 2013 Bisphenol A exposure is associated with low-grade urinary albumin excretion in children of the United States. Kidney International 83 741-748. (https://doi. org/10.1038/ki.2012.422)

Trasande L, Sathyanarayana S \& Trachtman H 2014 Dietary phthalates and low-grade albuminuria in US children and adolescents. Clinical Journal of the American Society of Nephrology 9 100-109. (https://doi. org/10.2215/CJN.04570413)

Warnatsch A, Ioannou M, Wang Q \& Papayannopoulos V 2015 Inflammation. Neutrophil extracellular traps license macrophages for cytokine production in atherosclerosis. Science 349 316-320. (https:// doi.org/10.1126/science.aaa8064)

Welsh GI \& Saleem MA 2011 The podocyte cytoskeleton - key to a functioning glomerulus in health and disease. Nature Reviews Nephrology 8 14-21. (https://doi.org/10.1038/nrneph.2011.151)

Received in final form 11 February 2019

Accepted 22 February 2019

Accepted Preprint published online 22 February 2019 https://joe.bioscientifica.com https://doi.org/10.1530/JOE-18-0608 (c) 2019 Society for Endocrinology Published by Bioscientifica Ltd. Printed in Great Britain 